\title{
Original
}

\section{Stainless and Titanium Fibers as Non-degradable Three-dimensional Scaffolds for Bone Reconstruction}

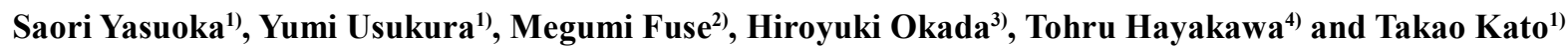 \\ 1) Department of Oral Implantology, Nihon University School of Dentistry at Matsudo, Matsudo, Japan \\ 2) Department of Laboratory Medicine for Dentistry, Nihon University School of Dentistry at Matsudo, Matsudo, Japan \\ 3) Department of Histology, Nihon University School of Dentistry at Matsudo, Matsudo, Japan \\ 4) Department of Dental Engineering, Tsurumi University School of Dental Medicine, Yokohama, Japan \\ (Accepted for publication, July 23, 2014)
}

\begin{abstract}
Stainless steel is used for various medical devices. Titanium is also widely used because of its tight bonding to bone tissue, namely osseointegration. The aim of the present study was to investigate the efficacy of three-dimensional stainless steel and titanium porous structure scaffolds by in vitro and in vivo experiments. Stainless steel and titanium fiber materials were designed as non-degradable metallic three-dimensional scaffolds with a three-dimensional porous structure for bone reconstruction. In one in vitro experiment, stainless steel and titanium disks were immersed in simulated body fluid. After immersion, a greater amount of hydroxyapatite precipitation was observed on titanium disks than on stainless steel disks. The crystallographic structure of precipitated hydroxyapatite was confirmed by the measurement of X-ray diffraction patterns. In another in vitro study, the adsorption behavior of fibronectin to stainless steel and titanium was monitored by the quartz crystal microbalance (QCM) method, which demonstrated more fibronectin adsorption onto titanium than onto stainless steel. In an in vivo experiment, three-dimensional stainless steel or titanium fibers were implanted into the cortical bone of the tibia of rabbits. Histological and histomorphometrical evaluation revealed a significantly greater amount of bone formation inside the porous area of the titanium fibers after 4 weeks of implantation. The present study also revealed better osteoconductivity of titanium than stainless steel. It is presumed that this was due to the differences in the characteristics of passive film between titanium and stainless steel. Titanium fibers are expected to be useful as a non-resorbable three-dimensional scaffold in bone reconstruction.
\end{abstract}

Key words: Titanium, Stainless steel, Bone formation, Simulated body fluid, Fiber

\section{Introduction}

Three-dimensional scaffolds have been widely used for bone reconstruction. Degradable materials, ceramics, such as apatite or tricalcium phosphate; polymers, such as polylactic acid or poly(lactide-glycolide); and their composites, such as apatite/ PLGA, are mainly applied ${ }^{1-3)}$, but these materials have the disadvantage of unacceptable mechanical properties, namely brittleness or easy transformation. The ability to form the required shape is also insufficient.

To improve mechanical and handling properties, nondegradable metallic three-dimensional scaffolds are expected to be another candidate for bone reconstruction. Among metallic materials, stainless steel and titanium are now commonly used for orthopedic and dental treatments. Stainless steel has been used

Correspondence to: Dr. Saori Yasuoka, Department of Oral Implantology, Nihon University School of Dentistry at Matsudo, 2-870-1 Sakaecho Nishi, Matsudo, Chiba, 271-8587 Japan; Tel.: +81-47-360-9633; Fax: +81-47-360-9633; E-mail: yasuoka.saori@nihon-u.ac.jp for medical and surgical devices, artificial joints and bone fixation plates or pins after bone fracture, stents for cardiovascular medicine, and wire and brackets for orthodontic treatments etc. ${ }^{4-}$ ${ }^{6}$, and titanium is now used as a dental implant material ${ }^{7}$.

Ganser et al. ${ }^{8}$ reported the clinical benefits of stainless steel and titanium for reducing pin track irritation/infection and pin loosening during external fracture fixation of the hind limbs of sheep, and found no clinically relevant substantial advantage in using either stainless steel or titanium pins. Choi et al. ${ }^{9)}$ performed a clinical comparative study between titanium and stainless steel for microsurgical cerebral aneurysm clip implants. They also reported no significant clinical outcome differences.

Therefore, we designed stainless steel and titanium fiber materials as non-degradable metallic three-dimensional scaffolds for bone reconstruction. First, in vitro apatite precipitation by immersion experiments and protein adsorption experiments were evaluated to investigate the mechanism of bone formation to stainless steel and titanium. For immersion experiments, stainless 
J.Hard Tissue Biology Vol. 23(4):407-414, 2014

Table 1. Ion concentration in Hanks' balanced salt solution without organic species

\begin{tabular}{llllllllll}
\hline \multicolumn{10}{c}{ Ion } \\
\hline & $\mathrm{Na}^{+}$ & $\mathrm{K}^{+}$ & $\mathrm{Mg}^{2+}$ & $\mathrm{Ca}^{2+}$ & $\mathrm{Cl}^{-}$ & $\mathrm{HPO}_{4}^{2-}$ & $\mathrm{SO}_{4}^{2-}$ & $\mathrm{HCO}^{3-}$ \\
Concentration $(\mathrm{mM} / \mathrm{I})$ & 142 & 5.81 & 0.811 & 1.26 & 145 & 0.778 & 0.811 & 4.17 \\
\hline
\end{tabular}

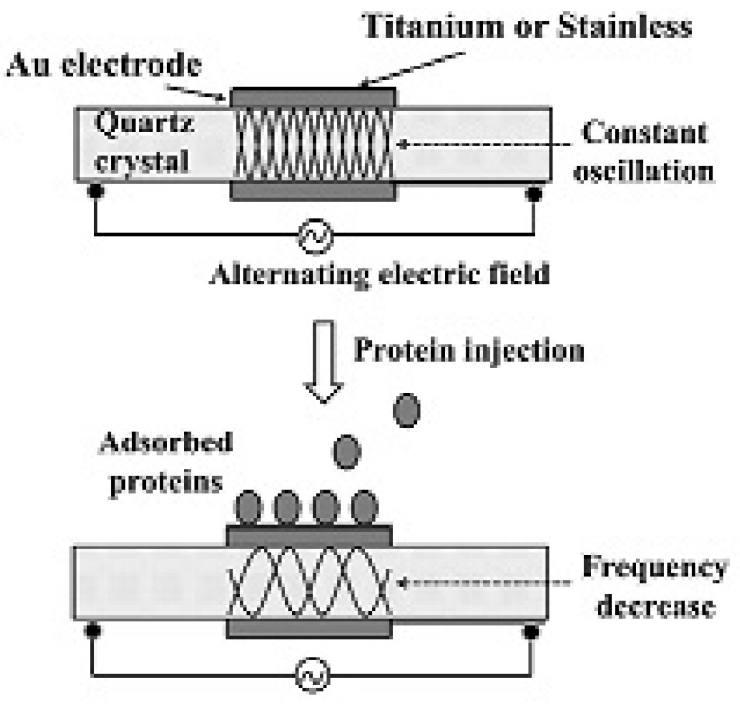

Figure 1. Principle of QCM

steel and titanium disks were employed instead of fibers because of the straightforward analysis of precipitated compounds. Adsorption experiments of fibronectin, a cell-adhesive protein, to stainless steel and titanium were also conducted. For protein adsorption experiments, the quartz crystal microbalance (QCM) method ${ }^{10)}$ was employed. Furthermore, in the present study, bone responses to stainless steel and titanium fibers were evaluated in vivo by implantation into the cortical bone of the tibiae of rabbits.

\section{Materials and Methods}

\section{Immersion in simulated body fluid}

As in vitro biocompatibility evaluation, apatite formation on stainless steel disks (St-disk; Furuuchi Chemical Corp., Tokyo, Japan) and titanium disks (Ti-disk; Furuuchi Chemical Corp., Tokyo, Japan) was monitored after immersion in simulated body fluid. St-disk and Ti-disk, $12 \mathrm{~mm}$ diameter and $1.0 \mathrm{~mm}$ thick, were immersed in $20 \mathrm{ml}$ simulated body fluid at $37{ }^{\circ} \mathrm{C}$ in sealed polystyrene bottles for 1 and 3 days. As simulated body fluid, Hanks' balanced salt solution (HBSS) without organic species $(\mathrm{pH}=7.4)$ was used ${ }^{11,12)}$. The ion concentrations of HBSS are listed in Table 1 . The solutions and bottles were changed every day to expose the specimens to fresh solutions. After immersion, St-disk and Ti-disk were rinsed with double-distilled water to remove HBSS solution and then dried in a desiccator. The surface of the samples before and after was examined by scanning electron microscope (SEM, S-2700; Hitachi, Tokyo, Japan) at an accelerating voltage of $15 \mathrm{kV}$. The specimens were coated with platinum.

The cross-sectional views of the precipitates on St-disk and Ti-disk were also observed to monitor the difference of precipitated amounts on each disk. After 3 days' immersion in HBSS, the specimens were embedded in polyester resin (Strus; Marumoto Strus Inc., Ltd. Tokyo, Japan). After curing the resin, the samples were cut vertically so that cross-sectional samples of the precipitates/St-disk or Ti-disk could be obtained. The cut samples were ground and then polished with diamond paste down to $0.25 \mu \mathrm{m}$. After ultrasonically cleaning, the samples were observed with SEM after platinum coating.

The crystallographic structure of precipitates on St-disk and Ti-disk was analyzed by X-ray diffraction (XRD, Multi Flex 2kW; Rigaku, Tokyo, Japan), which had an X-ray source of CuK and power of $50 \mathrm{kV} \times 50 \mathrm{~mA}$, and was also determined by Fouriertransform infrared spectroscopy (FT-IR, FTIR-8400S; Shimadzu Corp., Kyoto, Japan) by the KBR method. For FT-IR measurement, precipitated products were removed from St-disk or Ti-disk.

\section{Protein adsorption by quartz crystal microbalance (QCM) method}

The principle of QCM is depicted in Fig. $1^{10)}$. An AT-cut quartz crystal is sandwiched between gold ( $\mathrm{Au}$ ) electrodes. Application of an alternative electric current across the quartz results in thickness-shear mode oscillation of the quartz crystal. When a molecule binds to the oscillating quartz crystal, the oscillating frequency decreases in relation to the amount of protein bound to the crystal surface. The amount of protein adsorbed onto the biomaterial can be estimated by the Sauerbrey equation ${ }^{13)}$.

$$
\Delta F=-\frac{2 F_{0}{ }^{2} \Delta m}{A \sqrt{\rho_{q} \mu_{q}}}
$$

Where $\Delta \mathrm{F}$ is the measured frequency shift $(\mathrm{Hz}), \Delta \mathrm{m}$ is the mass change $(\mathrm{g}), \mathrm{F} 0$ is the fundamental frequency of the quartz crystal $(27 \times 106 \mathrm{~Hz}), \mathrm{A}$ is the electrode area $\left(0.049 \mathrm{~cm}^{2}\right), \rho q$ is the density of quartz $\left(2.65 \mathrm{gcm}^{-3}\right)$, and $\mu \mathrm{q}$ is the shear modulus of quartz $\left(2.95 \times 1011 \mathrm{dyncm}^{-2}\right)$.

The frequency changes were monitored on a personal computer. The QCM apparatus used was a $27 \mathrm{MHz}$ AFFINIX $\mathrm{QN} \mu$ (Initium Co., Ltd, Tokyo, Japan) with $500 \mu \mathrm{l}$ cells and equipped with a temperature control system and stirring bar. The temperature was maintained at $25 \pm 1{ }^{\circ} \mathrm{C}$ and the solution in the cells was stirred during the measurements. The surface area of the 
Saori Yasuoka et al.: Stainless and Titanium Fibers for Bone Reconstruction
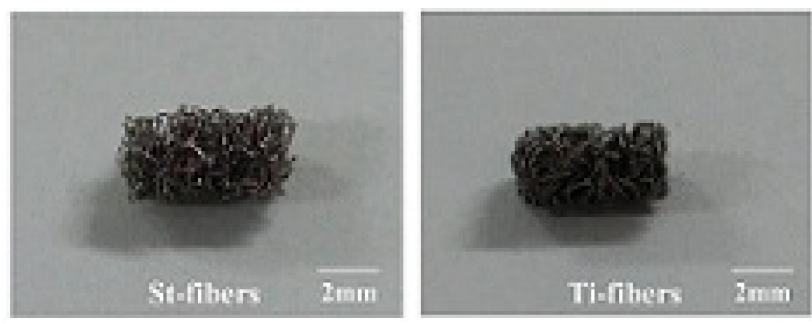

Figure 2. Macroscopic appearances of St-fibers and Ti-fibers
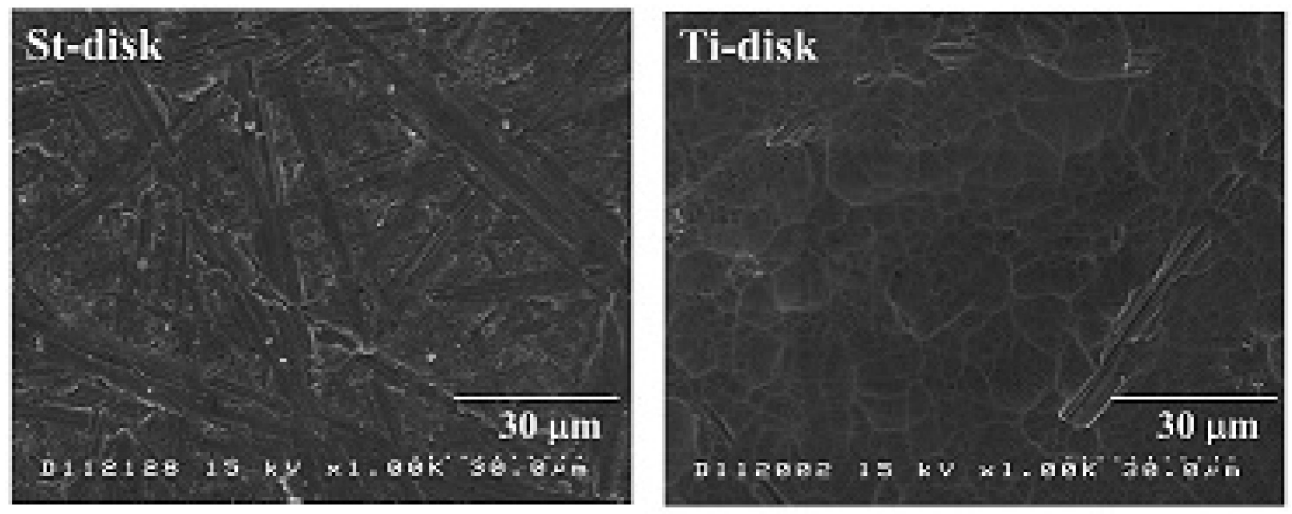

Figure 3. SEM images of St-disks and Ti-disks before immersion in HBSS

$\mathrm{Au}$ electrode was $4.9 \mathrm{~mm}^{2}$. The stainless sensor (St-sensor) and titanium sensor (Ti-sensor) were prepared by sputter coating stainless steel or titanium onto Au electrodes. A stainless steel or titanium disk (99.99 mass \%; ULVAC, Inc., Kanagawa, Japan) was used as the target. Using Sputtering Deposition Equipment (CS200; ULVAC, Inc., Chigasaki, Japan), stainless steel or titanium was sputter coated at a pressure of $0.2 \mathrm{~Pa}$ for $30 \mathrm{~min}$.

Significant differences were determined using GraphPad software (Graphpad Prism; GraphPad Software Inc., San Diego, CA, USA). Statistical significance was set at $\mathrm{p}<0.05$.

\section{Stainless steel fibers (St-fibers) and titanium fibers (Ti-fibers)}

Cylindrical stainless steel fibers (St-fiber) and titanium fibers (Ti-fiber) (Fig. 2; HI-LEX Corp., Hyogo, Japan) with a volumetric porosity of $87 \%$ were used. The fiber diameter of St-fiber was 80 $\mu \mathrm{m}$ and that of Ti-fiber was $85 \mu \mathrm{m}$. The diameter of cylindrical Stfibers and Ti-fibers was $2.8 \mathrm{~mm}$ and the length was $5.0 \mathrm{~mm}$. Stfibers and Ti-fibers were constructed by intertwining multiple thin stainless steel and titanium fibers, respectively. This results in a very porous interconnected structure.

The fiber structure of St-fibers and Ti-fibers was confirmed by SEM at an accelerating voltage of $15 \mathrm{kV}$.

\section{Implantation of Scaffolds (St-fibers and T-fibers)}

The animal study was conducted in accordance with the animal experimental ethics guidelines of Nihon University School of Dentistry at Matsudo (Certificate Number: AP09MD019). Six 20week-old female Japanese White rabbits weighing approximately
$3.5 \mathrm{~kg}$ were used. The cylindrical fiber scaffolds were inserted into the cortical bone of rabbit tibiae according to a previously reported technique ${ }^{3)}$. Each animal received only one scaffold for animal welfare. Three St-fibers and three Ti-fibers were placed. Before surgery, all fiber scaffolds were sterilized in an autoclave.

Surgery was performed under general inhalation anesthesia with a $5 \%$ isoflurane and oxygen mixture, which was reduced to $2 \%$ isoflurane during surgical manipulation. Local anesthesia was

performed by xylocaine injection. The hind legs of the rabbits were shaved, washed and disinfected with iodine tincture.

A longitudinal incision was made on the medial surface of the left tibiae, and the medial surface of tibiae was exposed. After drilling a pilot hole with a diameter of $0.6 \mathrm{~mm}$, the hole was gradually widened with different drills to the final diameter, 2.8 $\mathrm{mm}$, of the scaffold. The bone defect was prepared with a very gentle surgical technique using a low rotational drilling speed (500 rpm) and continuous internal cooling. After insertion of the scaffolds, the soft tissues were closed in separate layers using absorbable silk 4-0 sutures (absorbable Vicryl 3-0 sutures). To reduce the perioperative infection risk, a prophylactic antibiotic, Shiomalin ${ }^{\circledR}$ (equivalent to xLatamoxef Sodium), was administered postoperatively by subcutaneous injection.

Postoperatively, the animals were placed in a standard cage. They were fed water and rabbit diet ad libitum, and were allowed to move unrestricted at all times. The rabbits were sacrificed by inhalation of carbon dioxide 4 weeks after implantation of scaffolds.

\section{Histological procedures and evaluation}

The scaffolds and surrounding bone were excised immediately after sacrifice. Then, excess tissue of the excised tibiae was removed. Following fixation in $10 \%$ buffered formalin solution, the specimens were prepared for histological evaluation. Scaffolds containing tissue blocks were dehydrated through a graded series of ethanol and embedded in methylmethacrylate. After polymerization, non-decalcified thin sections were prepared using 

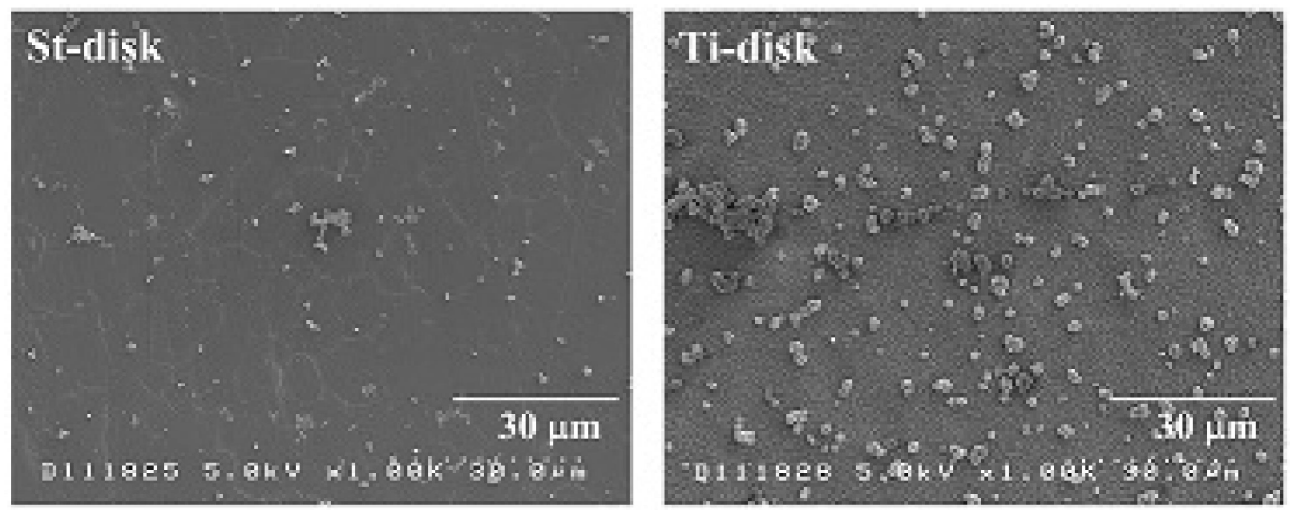

Figure 4. SEM images of St-disks and Ti-disks after 1 days immersion in HBSS
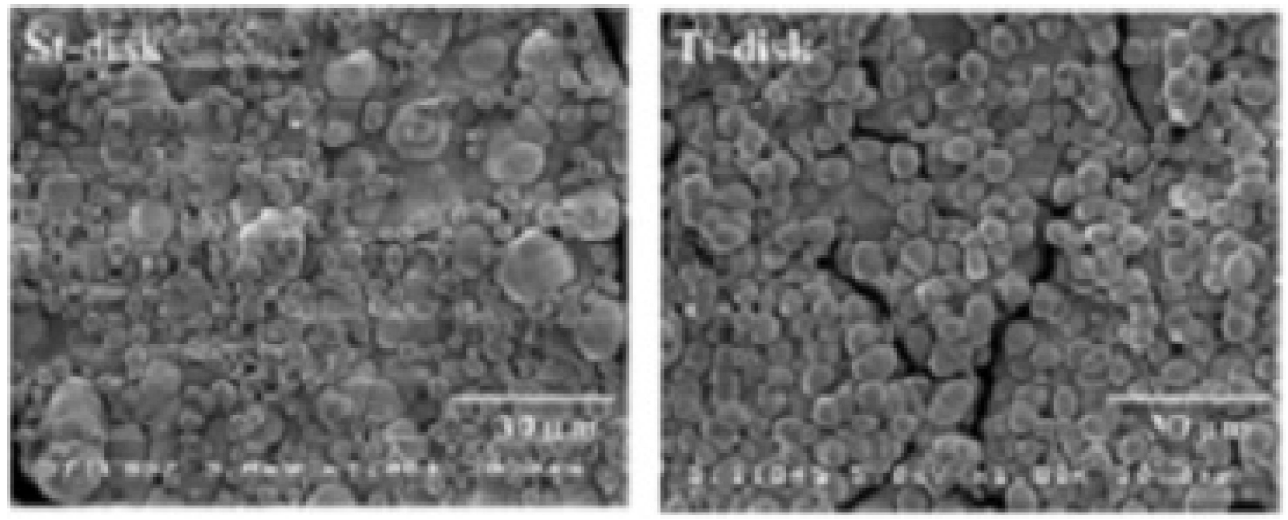

Figure 5. SEM images of St-disks and Ti-disks after 3 days immersion in HBSS
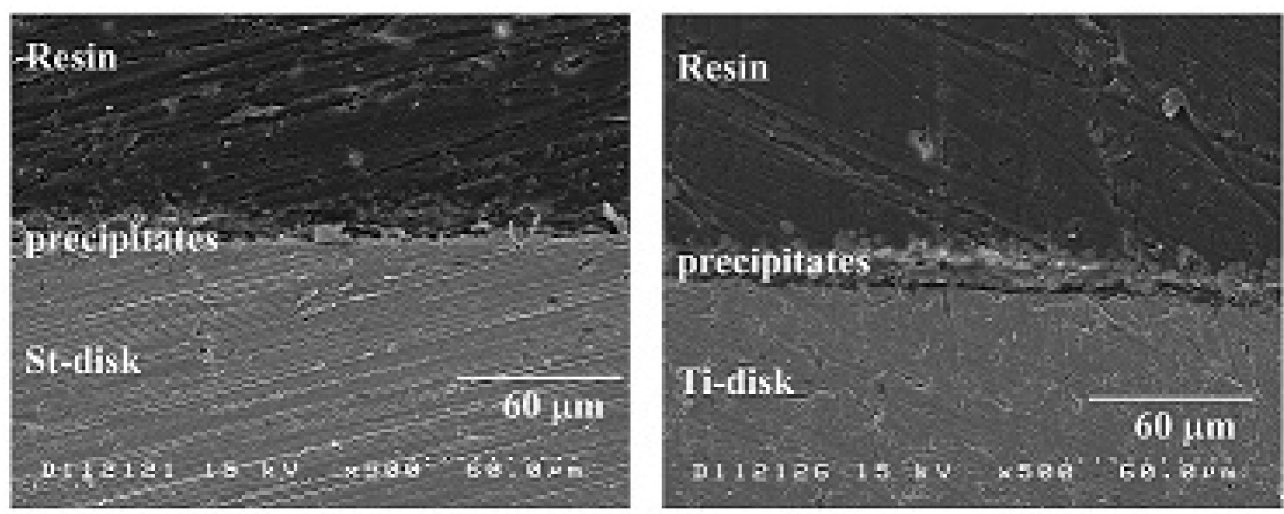

Figure 6. SEM images of cross-sectional views of St-disks and Ti-disks after 3 days immersion in HBSS

a cutting-grinding technique (EXAKT-Cutting Grinding System, BS-300Cp band system and $400 \mathrm{CS}$ micro grinding system; EXAKT, Norderstedt, Germany) ${ }^{14)}$. Sections were made in a transverse direction perpendicular to the axis of the tibiae, and were stained with methylene blue and basic fuchsine. Bone formation inside the St-fibers and Ti-fibers was evaluated using a light microscope (ECLIPSE 80i; Nikon Corporation, Tokyo, Japan; magnification $200 \times$ ) using an image analysis system (WinROOF; Visual System Division, Mitami Corporation, Tokyo, Japan).

\section{Result}

\section{Immersion in simulated body fluid}

Fig. 3 shows SEM images of the surface appearances of Stdisk and Ti-disk before HBSS immersion. Both had flat surfaces. Some grooves were prepared artificially.

Fig. 4 shows SEM images of the surfaces of St-disk and Tidisk after 1 day immersion in HBSS. Greater formation of white precipitates on Ti-disk was observed than on St-disk. After 3 days immersion in HBSS, both St-disk and Ti-disk surfaces were completely covered with precipitate substances, as shown in Fig. 5. For Ti-disk, a greater number of smaller globular precipitates could be observed.

Fig. 6 shows SEM images of the cross-section view of Stdisk and Ti-disk after 3 days immersion in HBSS. More precipitates 
Saori Yasuoka et al:: Stainless and Titanium Fibers for Bone Reconstruction

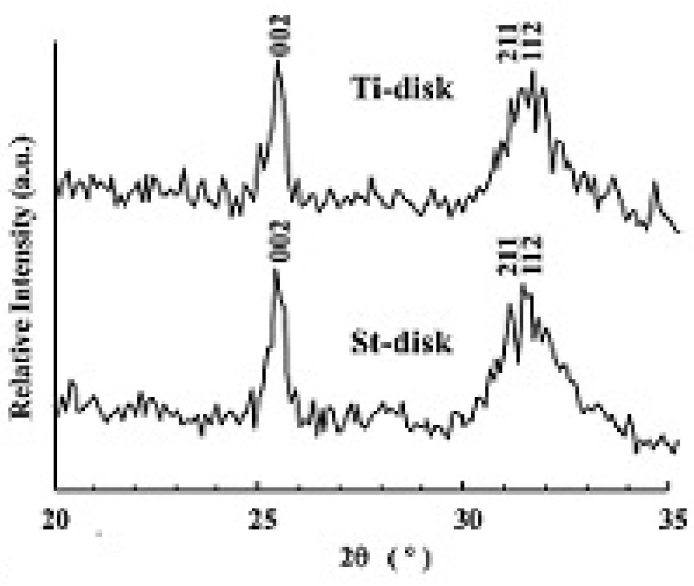

Figure 7. X-ray diffraction (XRD) patterns of white precipitates on St-disks and Ti-disks after 3-day immersion in HBSS

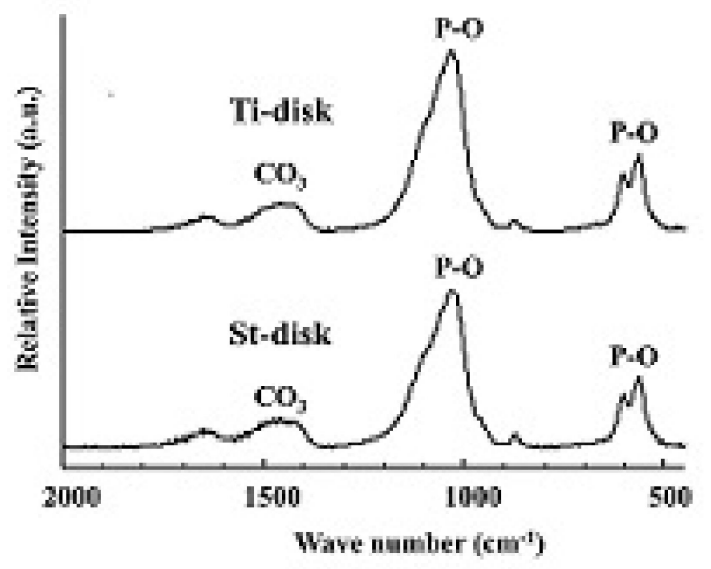

Figure 9. Frequency shift as a function of time for the adsorption of fibronectin to St-sensors and Ti-sensors.

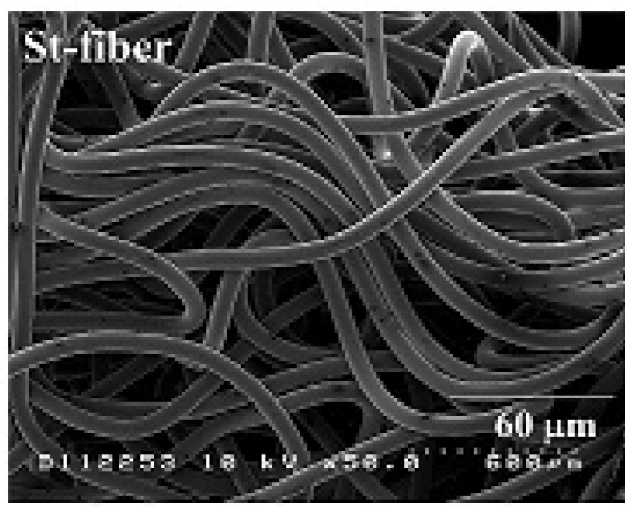

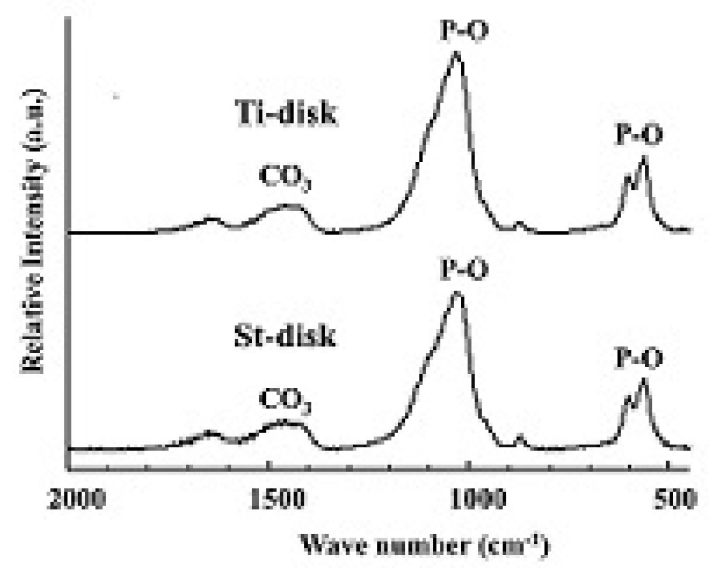

Figure 8. Fourier transform infrared (FT-IR) spectra of white precipitates on St-disks and Ti-disks after 3-day immersion in HBSS

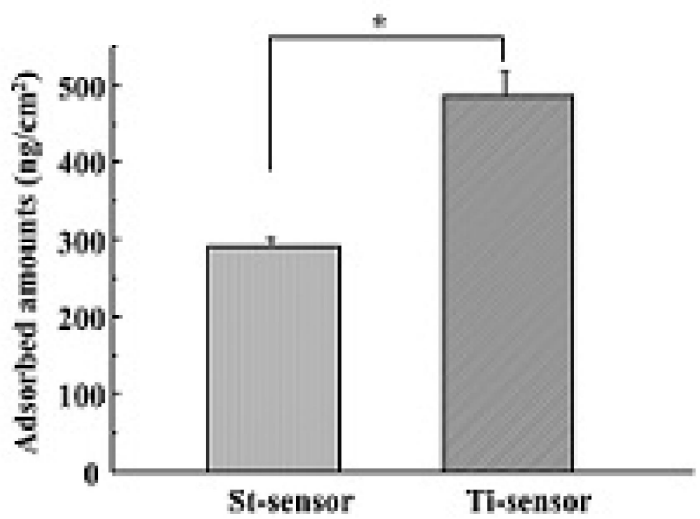

Figure 10. Estimated adsorbed amounts of albumin, fibronectin and collagen to titanium. $* \mathrm{p}<0.05$

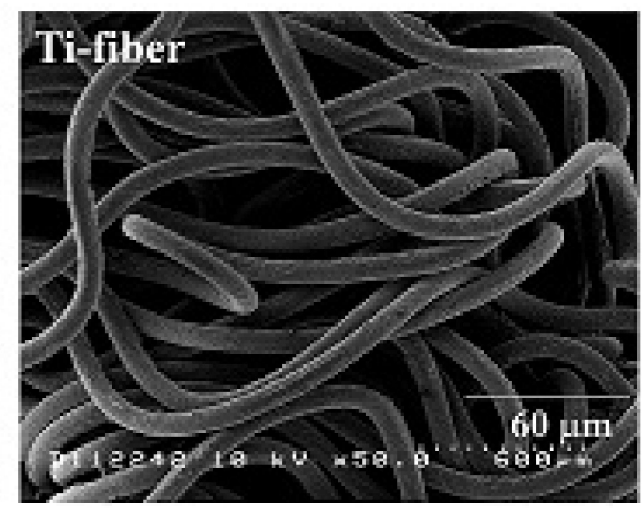

Figure 11. SEM of inner structures of St-fibers and Ti-fibers

were observed on Ti-disk than on St-disk. The thickness of precipitates on Ti-disk was approximately $10 \mu \mathrm{m}$ and was twice of that on St-disk.

XRD spectra demonstrated hydroxyapatite precipitation on St-disk and Ti-disk after 3 days immersion in HBSS, as shown in Fig. 7. Both immersed samples showed similar XRD patterns.
The peaks around $2=25-25.5^{\circ}$ and $2=32^{\circ}$ could be assigned as $002,211,112$ peaks of hydroxyapatite.

Fig. 8 shows the FT-IR spectrum of white precipitates on Stdisk and Ti-disk after 3 days immersion in HBSS. Both samples showed similar FT-IR spectra. There were two clusters of peaks, at $550 \sim 600 \mathrm{~cm}^{-1}$ and at $\sim 1050 \mathrm{~cm}^{-1}$. The former cluster was 

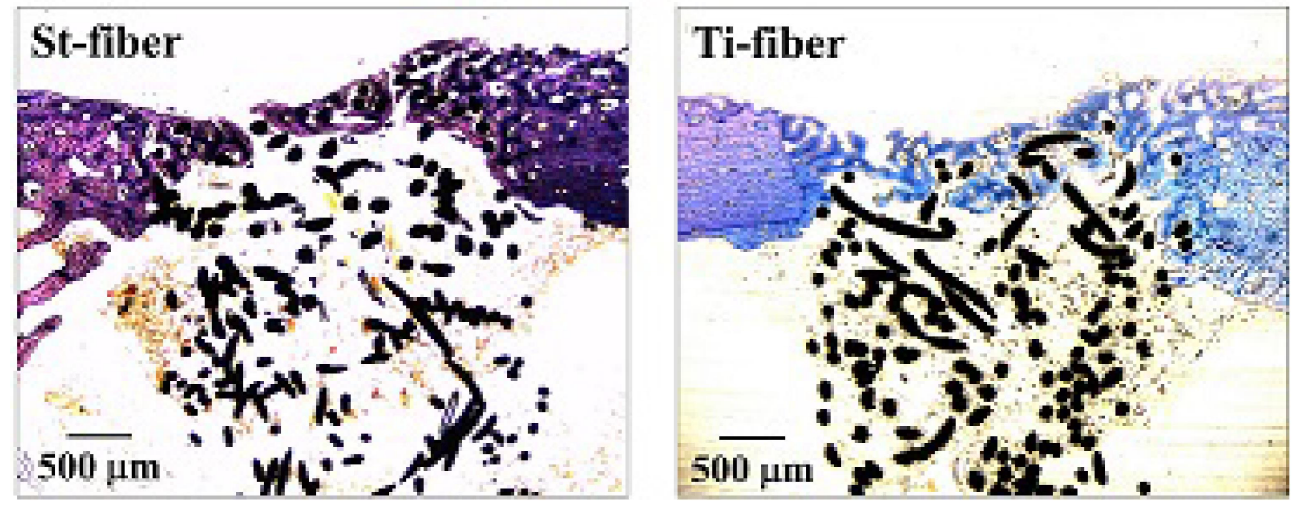

Figure 12. Histological appearances of St-fibers and Ti-fibers after 12 weeks' implantation. More bone formation was recognized in the space of Ti-fibers.

Table 2. Bone ingrowth percentage into the space of fiber structures

\begin{tabular}{ll}
\hline Sample & Bone ingrowth percentage (\%) \\
St-fibers & $23.7(3.5)$ \\
Ti-fibers & $37.9(8.1)$ \\
\hline
\end{tabular}

( ): standard deviation Significant differences between mean values $(\mathrm{p}<0.05)$

assigned as the $\mathrm{P}-\mathrm{O}$ bending mode and the latter as the $\mathrm{P}-\mathrm{O}$ stretching mode. These are attributed to the P-O bonds of hydroxyapatite. Peaks derived from carbonate groups were also observed $\sim 1450 \mathrm{~cm}^{-1}$, indicating that these carbonate groups were incorporated into the apatite structure. Thus, precipitated materials on St-disk and Ti-disk after immersion in HBSS could be regarded as hydroxyapatite.

\section{QCM measurement}

Fig. 9 shows the typical frequency shifts of the adsorption of fibronectin to St-sensor and Ti-sensor by QCM measurements. An immediate frequency decrease was observed after the injection of fibronectin. This rapid decrease in frequency is related to the rapid adsorption of proteins. At 30 min after the injection of fibronectin, the decrease of the frequency shift had almost reached a plateau. The decreased frequency shift of fibronectin adsorption to Ti-sensor was larger than that to St-sensor.

Fig. 10 shows the estimated amounts of proteins adsorbed on the St-sensor and Ti-sensor by the Sauerbrey equation ${ }^{13)}$. There was a significant difference in the adsorbed amounts of fibronectin between St-sensor and Ti-sensor $(\mathrm{p}<0.05)$.

\section{Bone formation after implantation}

SEM views of the fiber structure of St-fibers and Ti-fibers are shown in Fig. 11. The three-dimensional fiber structures were clearly identified for both fiber samples.

During the test period, the experimental animals remained in good health. At sacrifice, no clinical signs of inflammation or adverse tissue reactions were seen.
Histological appearances after 4 weeks' implantation are shown in Fig. 12. The overall cortical bone responses to St-fibers and Ti-fibers were similar and no clear appearances of macrophages or foreign-body giant cells were recognized in any specimen. Bone remodeling proceeded and no new bone formation was observed in bone marrow spaces. More bone ingrowth in the fiber structure was observed for Ti-fibers.

Table 2 shows the percentage of bone ingrowth in the space of the fiber structure of St-fibers and Ti-fibers. Ti-fibers provided significantly more bone ingrowth into the space than St-fibers ( $p$ $<0.05)$.

\section{Discussion}

In the present study, the efficacy of St-fibers and Ti-fibers as three-dimensional scaffolds for bone regeneration was evaluated by in vitro immersion and QCM experiments and in vivo implantation experiments in bone defects of rabbits.

Immersion experiments in HBSS revealed that more apatite precipitated onto Ti-disks than St-disks, and QCM measurement demonstrated greater fibronectin adsorption onto titanium than stainless steel. Animal experiments revealed that more bone ingrowth was obtained with Ti-fibers than St-fibers.

It is generally recognized that the in vivo osteoconductivity of biomaterials is mirrored by their in vitro apatite precipitation ability in simulated body fluid ${ }^{15)}$. Tanimto et al. ${ }^{16)}$ demonstrated that in vivo bone formation of sintered tricalcium sheets confirmed the results of in vitro HBSS immersion experiments. Mochizuki et al. ${ }^{17)}$ and Hayakawa et al..$^{3)}$ investigated the relationship between the crystallinity of apatite and bone formation. Both reported that apatite composite with low crystallinity produced greater new bone formation after implantation into the cortical bone of rabbit tibiae and also induced more rapid and greater amounts of hydroxyapatite precipitation after immersion in HBSS. Our in vivo and in vitro experiments corresponded with the above previous results.

The adsorption of body-fluid proteins, including extracellular matrix components at the implant-tissue interface, is the first 
Saori Yasuoka et al.: Stainless and Titanium Fibers for Bone Reconstruction

biological response to titanium after implantation. The initial adsorption of proteins is known to affect some biological responses, such as cell proliferation and growth, and microbial colonization $^{18,21)}$. In the present study, the adsorption behavior of fibronectin to stainless steel and titanium was monitored by the QCM method, a simple technique to detect molecular behavior on a surface. Okahata et al. ${ }^{20,21)}$ reported a pioneering study using the QCM technique to detect the interaction of various biomolecules, such as deoxyribonucleic acid (DNA), peptide and carbohydrate. Hayakawa et al. ${ }^{22)}$ also monitored the complex formation between DNA and basic fibroblast growth factor using the QCM method. Yoshinari et al. ${ }^{23)}$ reported that the QCM technique was useful for determining the adsorption behavior of simvastatin acid or titanium binding peptide to the titanium surface.

In the present study, 27MHz QCM was employed. The higher fundamental frequency QCM produces higher sensitivity. It is recommended that a $27 \mathrm{MHz}$ QCM with a very low noise level of $\pm 0.05 \mathrm{~Hz}$ is useful for detecting the reactions of biomolecules ${ }^{24,25}$. Hayakawa evaluated the adsorption behaviors of fibronectin and collagen to a gold sensor ${ }^{26)}$.

Fibronectin is a well-known cell-adhesive protein and is believed to have an important role in governing the interactions of implants with their matrices ${ }^{27)}$. The present QCM measurement clearly revealed the difference in the adsorbed amounts of fibronectin to titanium and stainless steel. It is presumed that greater amounts of fibronectin adsorption onto titanium will enhance cell attachment and proliferation, which will provide a better bone response.

It is suggested that more apatite precipitation onto Ti-disks compared to St-disks and greater fibronectin adsorption onto titanium than stainless steel provided more bone ingrowth with Ti-fibers than St-fibers. The present study clearly revealed the better osteoconductivity of titanium than stainless steel by both in vitro and in vivo experiments.

Both titanium and stainless steel are non-precious metals and have a surface oxide film, named a passive film. It is well known that a passive film on metallic materials plays an important role in corrosion resistance ${ }^{28}$. Although the detailed reason for the difference obtained in the present study between titanium and stainless steel is not clear, it is presumed that the difference in the characteristics of passive film between titanium and stainless steel will cause different bone responses and/or protein adsorption.

Hanawa et al. ${ }^{29)}$ characterized the surface oxide film of stainless steel after immersion in HBSS by X-ray photoelectron spectroscopy. It revealed that the thickness of surface oxide film

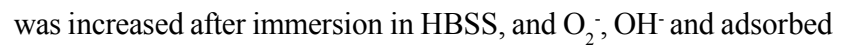
water were present in the oxide surface film of stainless steel. Dehydration and oxidation ocurred by immersion in HBSS and resulted in an increased concentration of $\mathrm{O}^{2-}$ and decreased concentration of $\mathrm{OH}^{-}$.
In contrast, titanium has both basic and acidic hydroxyl groups in the oxide film ${ }^{30)}$. It is presumed that the basic hydroxyl group, which is negatively charged, will play a significant role in attracting calcium ions in HBSS or body fluid and will provide a better bone response.

Delmi et al. ${ }^{31)}$ reported that the adsorption of radiolabelled fibronectin was found to be significantly greater on titanium coverslips than on stainless steel coverslips. Their results accorded with the present results. It is well known that many factors, such as electric interaction and hydrophobic-hydrophilic interaction, affect protein adsorption onto metallic materials. The reason for the greater fibronectin adsorption onto titanium than stainless steel will be further studied in detail.

St-fibers and Ti-fibers have a three-dimensional porous structure and are non-resorbable materials. Regarding clinical implant treatment, it is expected that non-degradable St-fibers or Ti-fibers will be useful as a scaffold for implant placement because of their better mechanical properties compared with degradable polymers or ceramics. It can be speculated that bone ingrowth into the fiber structure of St-fibers or Ti-fibers will enhance the support of the implant due to bone integration into threedimensional porous scaffolds.

Finally, in the comparison between St-fibers and Ti-fibers, Ti-fibers showed superior bone ingrowth and the efficacy of Tifibers was suggested as a scaffold for bone regeneration. However, the present bone ingrowth percentage into Ti-fibers is not so high. In the meantime, some surface modifications, such as thin apatite coating or cell adhesive immobilization, is expected to produce greater bone ingrowth. More detailed studies of the clinical application of St-fibers or Ti-fibers will be performed in the next series of studies.

\section{Acknowledgements}

We are grateful to Associate Professor Takeshi Toyama, Department of Materials and Applied Chemistry, College of Science and Technology, Nihon University, for his help with the measurements of X-ray diffraction patterns and Fourier-transform infrared spectroscopy.

\section{References}

1. Liu X and Ma PX. Polymeric scaffolds for bone tissue engineering. Ann Biomed Eng 32: 477-486, 2004

2. Yoshikawa H, Tamai N, Murase T and Myoui A. Interconnected porous hydroxyapatite ceramics for bone tissue engineering. J R Soc Interface. 6: S341-S348, 2009

3. Hayakawa T, Mochizuki C, Hara H, Yang F, Shen H, Wang $\mathrm{S}$ and Sato M. In vivo evaluation of composites of PLGA and apatite with two different levels of crystallinity. J Mater Sci Mater Med 21: 251-258, 2010

4. Lemons JE and Phillips RW. Biomaterials for dental 
implants. In: Contemporary Implant Dentistry, edited by Misch CE, Mosby-Year Book, Inc., St. Louis, MO, 1993, pp259-278.

5. Disegi JA and Eschbach L. Stainless steel in bone surgery. Injury, Int J Care Injured 31 Suppl 4: 2-6, 2000

6. Hanawa T. Materials for metallic stents. J Artif Organs 12: 73-79, 2009

7. Hanawa T. A comprehensive review of techniques for biofunctionalization of titanium. J Periodont Implant Sci 41: 263-272, 2011

8. Ganser A, Thompson RE, Tami I, Neuhoff D, Steiner A and Ito K. An in vivo experimental comparison of stainless steel and titanium Schanz screws for external fixation. Eur J Trauma Emerg Surg 33: 59-68, 2007

9. Choi SH, Park CW, Kim YB, Kim EU, You CJ and Kim WK. The comparative study of microsurgical cerebral aneurysm clip implants; titanium clip vs. stainless steel clip. J Cerebrovasc Endovasc Neurosurg 14: 79-83, 2012

10. Okahata $\mathrm{Y}$ and Furusawa H. Gravimetry of biomolecules at the water-substrate interface -Quartz-crystal maicrobalance. J Surf Sci Soc Jpn 25: 131-138, 2004

11. Hanawa T, Kon M, Ukai H, Murakami K, Miyamoto Y and Asaoka K. Surface modification of titanium in calcium-ioncontaining solutions. J Biomed Mater Res 34: 273-278, 1997

12. Hanawa $\mathrm{T}$ and Ota M. Calcium phosphate naturally formed on titanium in electrolyte solution. Biomaterials 12: 767774,1991

13. Sauerbrey G. Verwendung von Schwingquarzen zur Wägung dünner Schichten und zur Mikrowägung. Z Phys 155: 206222,1959

14. Donath K and Breuner G. A method for the study of undecalcified bones and teeth with attached soft tissues. The Sage-Schliff (sawing and grinding) technique. J Oral Pathol 11: 318-326, 1982

15. Kokubo $\mathrm{T}$ and Takadama $\mathrm{H}$. How useful is $\mathrm{SBF}$ in predicting in vivo bone bioactivity? Biomaterials 27: 2907-2915, 2006

16. Tanimoto Y, Hayakawa T, Sakae T and Nemoto K. Characterization and bioactivity of tape-cast and sintered TCP sheets. J Biomed Mater Res A 76: 571-579, 2006

17. Mochizuki C, Sasaki Y, Hara H, Sato M, Hayakawa T, Yang F, Hu X, Shen H and Wang S. Crystallinity control of apatite through Ca-EDTA complexes and porous composites with PLGA. J Biomed Mater Res B Appl Biomater 90: 290-301, 2009

18. Puleo DA and Nanci A. Understanding and controlling the bone-implant interface. Biomaterials 20: 2311-2321, 1999

19. Kasemo B and Lausmaa J. The biomaterial-tissue interface and its analogues insurfae science and technology. In: The Bone-Biomaterial Interface, edited by Davies JE, University of Toronto Press, Toronto, 1991, pp19-32.

20. Okahata Y, En-na G and Ebato H. Synthetic chemoreceptive membranes. Sensing bitter or odorous substances on a synthetic lipid multibilayer film by using quartz-crystal microbalances and electric responses. Anal Chem 62: 14311438,1990

21. Okahata $Y$, Masunaga $Y$, Matsuno $H$ and Furusawa $H$. Quantitative detection of a DNA ligase reaction on a quartzcrystal microbalance. Nucleic Acids Symp Ser 42: 147-148, 1999

22. Hayakawa T, Fukushima T, Kawaguchi M, Inoue Y, Taniguchi $\mathrm{K}$, Kaminishi H. Monitoring of DNA complex formation by means of quartz crystal microbalance. J Oral Tissue Engin 4: 61-67, 2006

23. Yoshinari M, Kato T, Matsuzaka K, Hayakawa T and Shiba K. Prevention of biofilm formation on titanium surfaces modified with conjugated molecules comprised of antimicrobial and titanium-binding peptides. Biofouling 26: 103-110, 2010.

24. Nihira T, Mizuno M, Tonozuka T, Sakano Y, Mori T and Okahata Y. Kinetic studies of site-directed mutational isomalto-dextranase-catalyzed hydrolytic reactions on a 27 MHz quartz-crystal microbalance. Biochemistry 44: 94569461, 2005

25. Furusawa $\mathrm{H}$, Takano $\mathrm{H}$ and Okahata $\mathrm{Y}$. Transient kinetic studies of pH-dependent hydrolyses by exo-type carboxypeptidase $\mathrm{P}$ on a $27-\mathrm{MHz}$ quartz crystal microbalance. Anal Chem 80: 1005-1011, 2008

26. Hayakawa T. Analysis of protein adsorption using 27-MHz quartz-crystal microbalance method. J Jpn Soc Den. Prod 26: $15-20,2012$

27. MacDonald DE, Markovic B, Allen M, Somasundaran P and Boskey AL. Surface analysis of human plasma fibronectin adsorbed to commercially pure titanium materials. J Biomed Mater Res 41: 120-130, 1998

28. Phillips RW. Tarnish and corrosion. In :Skinners's Science of Dental Materials, ed by Phillips RW, W. B. Saunders Company, Philadelphia, 1982, pp290-301.

29. Hanawa T, Hiromoto S, Yamamoto A, Kuroda D and Asami K. XPS characterization of the surface oxide film of $316 \mathrm{~L}$ stainless steel samples that were located in quasi-biological environments. Mater Trans 43: 3088-3092, 2002

30. Boehm HP. Acidic and basic properties of hydroxylated metal oxide surfaces. Faraday Discuss Faraday Soc 52: 264275,1971

31. Delmi M1, Vaudaux P, Lew DP and Vasey H. Role of fibronectin in staphylococcal adhesion to metallic surfaces used as models of orthopaedic devices. J Orthop Res 12: $432-438,1994$ 